\title{
EasyHalf - Uma Aplicação Web para Aprimoramento do Processo de Obtenção de Descontos de Meia-entrada
}

\author{
Vicente Terra $^{1}$, Ana Carolina G. Inocêncio ${ }^{2}$, Heitor A. X. Costa ${ }^{1}$, \\ Paulo Afonso Parreira Júnior ${ }^{1}$ \\ ${ }^{1}$ Departamento de Ciência da Computação - Universidade Federal de Lavras (UFLA) \\ Caixa Postal 3.037 - 37.200-900 - Lavras - MG - Brasil \\ ${ }^{2}$ Curso de Ciência da Computação - Universidade Federal de Jataí (UFJ) \\ 75.801-615 - Jataí - MG - Brasil \\ \{vicenteterra, anacarolina.inocencia\}@gmail.com, \\ \{heitor, pauloa.junior\}@ufla.br
}

\begin{abstract}
This work aims to improve the process of obtaining discounts known as "meia-entrada" granted to students, guaranteeing greater agility and reliability to this process. To accomplish that, a web application, called EasyHalf, was developed, which allows establishments to verify, in real time, the situation of a student's enrollment, by means of the registration number. After the development, the EasyHalf application was evaluated by students, in order to verify their usefulness and ease of use and the results were positive.
\end{abstract}

Resumo. Este trabalho visa melhorar o processo de obtenção de descontos conhecido como "meia-entrada" concedido aos alunos, garantindo maior agilidade e confiabilidade a esse processo. Para tanto, foi desenvolvido um aplicativo web, denominado EasyHalf, que permite que estabelecimentos verifiquem, em tempo real, a situação da matrícula de um aluno, por meio do número de matrícula. Após o desenvolvimento, a aplicação EasyHalf foi avaliada por estudantes, a fim de verificar sua utilidade e facilidade de uso e se os resultados foram positivos.

\section{Introdução}

\subsection{Contextualização e Motivação}

Segundo a Lei N 12.993 [República Federativa do Brasil, 2003], publicada em dezembro de 2013 e popularmente conhecida como "Lei da Meia-Entrada", é assegurado aos estudantes (e também aos jovens de baixa renda inscritos nos Programas Sociais do Governo Federal), o direito de acesso a alguns estabelecimentos e eventos, tais como cinemas, teatros, espetáculos musicais, entre outros, mediante o pagamento de metade do valor cobrado para o público em geral. Para que esse direito seja garantido, os estabelecimentos nos quais os indivíduos requerem o desconto exigem algum documento que comprove que eles estão realmente matriculados em uma instituição de ensino ou que estejam cadastrados em programas sociais governamentais. No caso dos estudantes, os documentos aceitos para comprovação pela maioria dos 
estabelecimentos, conforme especificado no texto da "Lei da Meia-Entrada", são os seguintes [República Federativa do Brasil, 2003]:

Carteira de Identificação Estudantil (CIE), emitida pela Associação Nacional de Pós-Graduandos (ANPG), pela União Nacional dos Estudantes (UNE), pela União Brasileira dos Estudantes Secundaristas (UBES), pelas entidades estaduais e municipais filiadas àquelas, pelos Diretórios Centrais dos Estudantes (DCEs) e pelos Centros e Diretórios Acadêmicos, com prazo de validade renovável a cada ano, conforme modelo único nacionalmente padronizado e publicamente disponibilizado pelas entidades nacionais antes referidas e pelo Instituto Nacional de Tecnologia da Informação (ITI), com certificação digital deste, podendo a carteira de identificação estudantil ter $50 \%$ (cinquenta por cento) de características locais.

Entretanto, há instituições de ensino em que os DCEs e Centros Acadêmicos não emitem Carteiras de Identificação Estudantil (CIE). Assim sendo, os estudantes precisam recorrer à UNE para adquiri-las, mediante o pagamento de uma taxa anual de R\$ 35,00 [UNE, 2018]. Os estudantes que, por não terem conhecimento desse serviço ou por não estarem dispostos a (não terem condições financeiras de) arcar com esses custos, utilizam a forma mais comum de se obter acesso ao desconto de meia-entrada, que é por meio da aquisição, manual ou eletronicamente, de um atestado de matrícula, emitido gratuitamente pela instituição de ensino à qual eles estão vinculados. Visto que muitos estabelecimentos exigem que os atestados de matrícula sejam recentes, essa estratégia possui algumas limitações, tais como:

1) é necessário que os estudantes retirem atestados de matrícula sempre que quiserem fazer uso do direito à meia-entrada, o que pode trazer transtornos e dificuldades, principalmente quando o processo para emissão/validação desses atestados é moroso. Há instituições de ensino, por exemplo, nas quais o estudante deve imprimir o atestado de matrícula e levá-lo à secretaria do seu respectivo curso para ser validado. Em outros casos, o representante do estabelecimento deve validar o atestado de matrícula por meio do portal acadêmico da instituição de ensino do estudante. Isso pode dificultar o trabalho dos funcionários do estabelecimento e reduzir a qualidade do atendimento aos clientes, uma vez que os portais acadêmicos das instituições de ensino são diversos e possuem formas de acesso e funcionamento diferentes; e

2) apesar de alguns estabelecimentos aceitarem uma versão digital do atestado de matrícula, muitos exigem a versão impressa, o que gera a necessidade da impressão de diversas cópias ao longo do ano.

Há estabelecimentos que aceitam atestados de matrícula mais antigos, porém essa estratégia mitiga a confiabilidade do processo de obtenção da meia-entrada. Por exemplo, é possível que um estudante apresente um atestado de matrícula em um estabelecimento, mesmo não tendo mais vínculo com a instituição de ensino (devido a trancamentos, desligamentos, entre outros motivos). O mesmo problema ocorre com relação às CIE, emitidas pela UNE (ou por outras entidades autorizadas, conforme apresentado anteriormente), uma vez que a validação da matrícula do estudante é feita uma única vez, no momento de emissão da carteira, e a mesma tem validade de 12 (doze) meses [UNE, 2018]. 


\subsection{Objetivos}

Esse trabalho tem como objetivo aprimorar o processo de obtenção do desconto de meia-entrada por parte dos estudantes, garantindo maior agilidade e confiabilidade a esse processo. Para alcançar esse objetivo, foi desenvolvida uma aplicação web, denominada de EasyHalf, que é capaz de verificar, em tempo real, a situação da matrícula de um estudante, no momento da solicitação do desconto. Para isso, basta que o estudante apresente ao representante do estabelecimento seu número de matrícula e um documento de identificação com foto (por exemplo, $\mathrm{RG}, \mathrm{CNH}$, entre outros). Em muitos casos, a própria carteira de identificação do estudante na instituição de ensino já possui tais informações, i.e., número de matrícula e foto (é importante ressaltar que a carteira de identificação do estudante não é aceita pelos estabelecimentos para oferecimento do desconto, uma vez ela não possui data de validade).

Como objetivos específicos deste trabalho, tem-se: (1) reduzir a emissão de cópias de atestados de matrículas e, consequentemente, o impacto ambiental provocado por esse procedimento; (2) tornar mais ágil e confiável o processo de verificação de matrícula de estudantes, por parte dos estabelecimentos; e (3) garantir maior facilidade de acesso ao direito à meia-entrada, por parte dos estudantes.

Considerando que as informações dos estudantes são pessoais e de caráter privado (tais como, nome completo e situação de matrícula), foi feita uma consulta à Pró-Reitoria de Graduação (PRG) da Universidade Federal de Lavras (UFLA) sobre como proceder frente a essa situação (a UFLA foi escolhida para essa consulta, pois os autores deste trabalho estão vinculados a essa instituição, o que facilitou a comunicação necessária com o órgão jurídico responsável). De acordo com esta pró-reitoria, a Procuradoria Geral (PG) da UFLA define uma série de condições para que esses dados possam ser disponibilizados de forma legal. Algumas das condições mais importantes são resumidas a seguir:

1) o estabelecimento que desejar ter acesso aos dados dos estudantes deverá, por meio de seu representante legal, estar devidamente cadastrado junto à instituição de ensino e aceitar um termo de responsabilidade, com respeito ao uso desses dados; e

2) o estudante deverá autorizar o acesso e a disponibilização de seus dados aos estabelecimentos de seu interesse e também terá que aceitar um termo de responsabilidade, com respeito às suas decisões.

Assim sendo, a aplicação EasyHalf foi desenvolvida levando-se em consideração os aspectos legais mencionados anteriormente. Mais detalhes sobre esse assunto são apresentados na Seção 3.

O restante deste trabalho está organizado da seguinte forma: na Seção 2, são descritas as pesquisas realizadas com os potenciais usuários da aplicação EasyHalf, com o intuito de levantar seus requisitos; a Seção 3 contém a descrição da funcionalidade da EasyHalf, bem como de sua arquitetura; na Seção 4, são apresentados os resultados da avaliação realizada com a aplicação; por fim, a Seção 5 apresenta as conclusões e as propostas de trabalhos futuros. 


\section{Análise de Viabilidade}

A primeira etapa deste trabalho consistiu na realização de uma pesquisa sobre a viabilidade do desenvolvimento da aplicação EasyHalf. Esta pesquisa foi realizada com os estudantes, os quais possuem o direito ao desconto de meia-entrada. Para isso, um questionário foi elaborado, composto de 6 (seis) questões de múltipla escolha. O questionário foi divulgado por meio de redes sociais e fóruns no AVA (Ambiente Virtual de Aprendizagem) da UFLA. O questionário obteve 133 (cento e trinta e três) respostas e ficou disponível para preenchimento de Outubro de 2016 à Março de 2017.

O objetivo da aplicação do questionário foi entender importantes aspectos sobre como os estudantes fazem uso do direito à meia-entrada. O primeiro ponto abordado no questionário foi a frequência com que os estudantes solicitam o desconto de meiaentrada, em qualquer tipo de estabelecimento. Na Figura 1 encontram-se os resultados para esse tipo de questionamento. Pode-se notar que mais de $60 \%$ dos 133 estudantes respondentes utilizam o desconto de meia-entrada com frequência mensal ou maior. Essa frequência elevada mostrou a importância de que o processo de concessão de descontos seja ágil e o mais simples possível.

A segunda pergunta feita no questionário visou a esclarecer em quais tipos de estabelecimentos os estudantes mais fazem os requerimentos de desconto de meiaentrada. Os resultados da Figura 2 mostram que a maior parte dos estudantes solicita o desconto no cinema local. Esses mesmos estudantes, também solicitam o desconto com frequência em eventos (shows, festas, etc) e em empresas de transporte público.

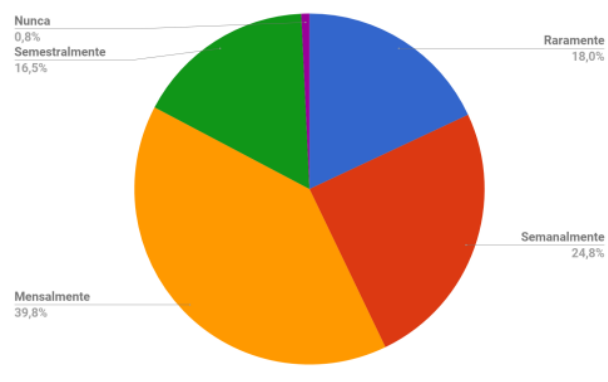

Figura 1. Frequência de uso do desconto de meia-entrada.

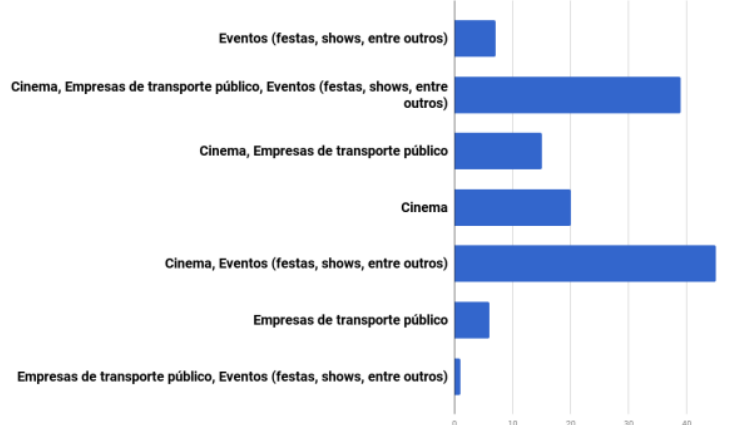

Figura 2.Estabelecimento onde os estudantes têm costume de solicitar o desconto de meia-entrada.

A terceira pergunta do questionário teve o intuito de descobrir qual era a forma de comprovação da situação da matrícula mais utilizada pelos estudantes, quando estes solicitam os descontos de meia-entrada. Como resultados, notou-se que grande parte dos estudantes (aproximadamente 82\%) comprova seu vínculo estudantil por meio de comprovantes de matrícula impressos, emitidos pela instituição de ensino, juntamente com um documento de identificação com foto. Já foram mencionados, na introdução deste trabalho, os problemas com essa forma de comprovação de matrícula, portanto os resultados dessa pergunta reforçam o fato de que é necessário um método de verificação de matrícula menos moroso.

Na quarta pergunta do questionário, buscou-se levantar os principais problemas encontrados pelos estudantes ao tentarem obter o desconto de meia-entrada nos 
estabelecimentos de interesse. A maior parte dos problemas (cerca de 95\%) foi causada pela data de validade vencida, ou pela ausência da mesma, no documento utilizado para comprovação de vínculo estudantil. Na quinta pergunta, buscou-se conhecer o que os estudantes achavam trabalhoso no processo já existente para comprovação da situação da matrícula. Os resultados dessa pergunta reafirmaram os problemas já discutidos na introdução deste trabalho. Grande parte dos estudantes (aproximadamente 90\%) respondeu que a necessidade de sempre portar um comprovante de matrícula atualizado é o que mais dificulta o acesso ao desconto de forma ágil e eficaz.

Na sexta e última questão, foi perguntado aos estudantes qual(is) documento(s) eles achavam ideal para portarem no momento da solicitação do desconto de meiaentrada. A maioria dos estudantes respondeu que o documento ideal seria a carteira de estudante emitida pela instituição de ensino. Em geral, este tipo de documento já é utilizado pelos estudantes em diversos setores da própria instituição de ensino, tais como a biblioteca e o restaurante universitário; este é o caso da UFLA.

\section{A Aplicação Easy Half}

Nesta seção, estão descritas a arquitetura e a funcionalidade da aplicação EasyHalf. Tal funcionalidade foi desenvolvida no intuito de atender às demandas levantadas pelos estudantes, conforme discutido na Seção 2. É importante ressaltar que a aplicação EasyHalf é software livre e seu código pode ser obtido em: https://github.com/VicenteTerra.

\subsection{Cadastro de Usuários}

A aplicação possui três tipos de usuários principais: (i) os estudantes, que solicitam o desconto de meia-entrada; (ii) os estabelecimentos, que consultam a situação da matrícula dos estudantes solicitantes do desconto; e (iii) as instituições de ensino, nas quais os estudantes estão matriculados e onde estão as informações sobre os mesmos. As instituições de ensino possuem a responsabilidade de autorizar ou não o acesso dos estabelecimentos aos dados de seus estudantes. Para isso, elas devem verificar a documentação enviada pelos estabelecimentos, conforme edital específico da própria instituição (isso será melhor detalhado adiante).

Para cada tipo de usuário, foi desenvolvida uma tela de cadastro na aplicação, solicitando as informações necessárias. Na Figura 3, vê-se a tela de cadastro de estudantes. Para concluir o cadastro, é necessário que o usuário aceite os termos e condições de uso da aplicação. Os termos e condições de uso para o estudante foram desenvolvidos com base nos requisitos legais, apontados pela PG da UFLA. Além dos campos básicos, necessários para o cadastro de estudantes, essa tela permite ao estudante visualizar a lista de estabelecimentos que sua instituição de ensino autorizou acesso aos seus dados. Para visualizar essa lista, o usuário deve clicar no botão "Lista de estabelecimentos", após selecionar a instituição desejada.

Assim como no caso dos estudantes, os estabelecimentos devem aceitar os termos e condições de uso da aplicação para concluir o cadastro. Além disso, os estabelecimentos devem fazer o upload dos documentos solicitados pelas instituições de ensino para que tenham seus cadastros validados. 


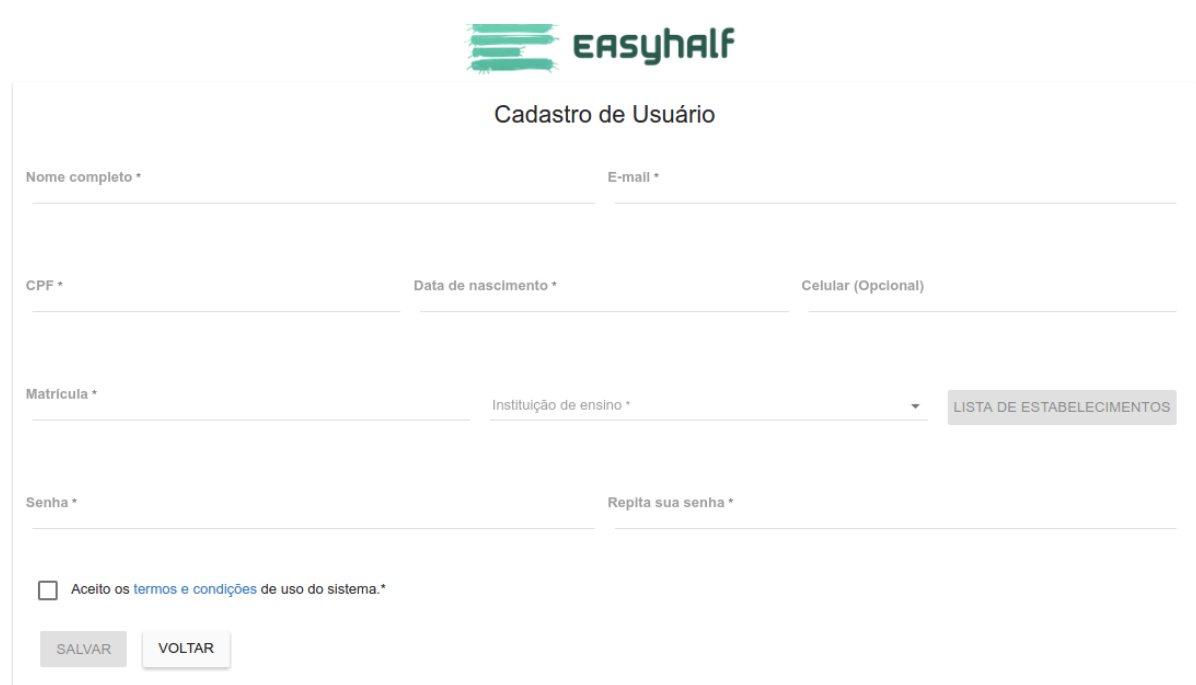

Figura 3. Tela de cadastro de estudantes.

\subsection{Verificação de Matrícula}

A principal funcionalidade da aplicação EasyHalf é a verificação da situação da matrícula dos estudantes solicitantes do desconto de meia-entrada. A aplicação permite que um estabelecimento, devidamente cadastrado e autenticado na aplicação, faça a consulta por meio do número de matrícula do estudante. Na Figura 4 é exibida a tela da aplicação, por meio da qual o estabelecimento poderá realizar tal consulta.

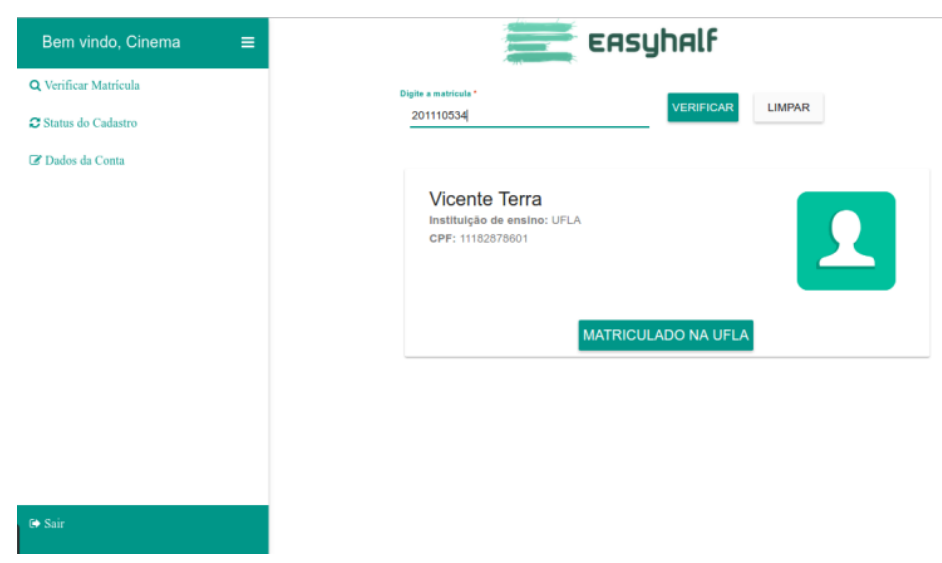

Figura 4. Resultado da verificação da situação da matrícula de um estudante.

A aplicação retornará informações básicas do estudante vinculado à matrícula consultada, tais como nome e instituição de ensino em que ele está matriculado. Tais informações são exibidas para confirmação da identidade do solicitante do desconto de meia-entrada. Além disso, a aplicação exibe a situação da matrícula no momento da consulta. Na Figura 4, pode-se visualizar o resultado de uma consulta: "Matriculado na UFLA". Neste exemplo, o estudante se encontra regularmente matriculado em uma instituição de ensino. Porém, a aplicação pode retornar outros três tipos de resultado: (i) um para o caso de o estudante estar vinculado a uma instituição, mas sua matrícula possuir algum tipo de impedimento, como por exemplo, matrícula trancada ou cancelada, entre outros; (ii) outro, no caso de o estudante não possuir vínculo com a instituição listada por ele no momento do cadastro; e (iii) o último, no caso de o 
estudante ter desativado sua conta na aplicação EasyHalf - conforme será explicado na Seção 3.3.

\subsection{Gerenciamento de conta}

Após o cadastro na aplicação, o usuário pode editar alguns dados de sua conta. Com isso, o usuário pode corrigir possíveis dados que tenham sido preenchidos incorretamente ou mesmo adicionar documentos que faltaram, no caso dos usuários do tipo "estabelecimento".

No caso dos estudantes, além de poder editar algumas de suas informações básicas, tais como nome, matrícula e instituição, em caso de mudanças de local de estudo, o mesmo poderá desativar sua conta. Ao fazer isso, os estabelecimentos não poderão consultar sua situação de matrícula, mesmo que sua instituição de ensino tenha autorizado tal estabelecimento para isso. Ao desativar sua conta, o estudante não poderá mais solicitar confirmação de matrícula por meio da aplicação EasyHalf. Tal funcionalidade foi criada para atender aos requisitos legais apontados pela PG da UFLA. Caso seja de interesse do estudante, ele poderá reativar sua conta a qualquer momento.

\subsection{Arquitetura da aplicação EasyHalf}

Uma preocupação ao se desenvolver a aplicação EasyHalf foi que ela fosse flexível a ponto de facilitar a inclusão de novos tipos de instituições de ensino. Isso, porque os estudantes matriculados em diferentes instituições de ensino são o principal públicoalvo da aplicação EasyHalf, e cada uma destas instituições possui diferentes sistemas de gestão acadêmica. EasyHalf foi desenvolvida utilizando a linguagem de programação Java [Java, 2019] no código backend e o framework Angular [Angular, 2019] no frontend. Além disso, sua arquitetura segue as boas práticas de desenvolvimento de software, com a utilização de design patterns, tais como AbstractFactory e Singleton (Gamma et al., 2000), entre outros.

A Figura 5 descreve a arquitetura da aplicação EasyHalf. O principal ponto a ser destacado desta arquitetura é a definição de uma interface Java, denominada "ConsultaMatricula", a qual deve ser implementada por classes que representam as instituições de ensino cadastradas no sistema. O objetivo dessa interface é prover um protocolo de comunicação comum entre a aplicação EasyHalf e os sistemas de gestão acadêmica das instituições de ensino.

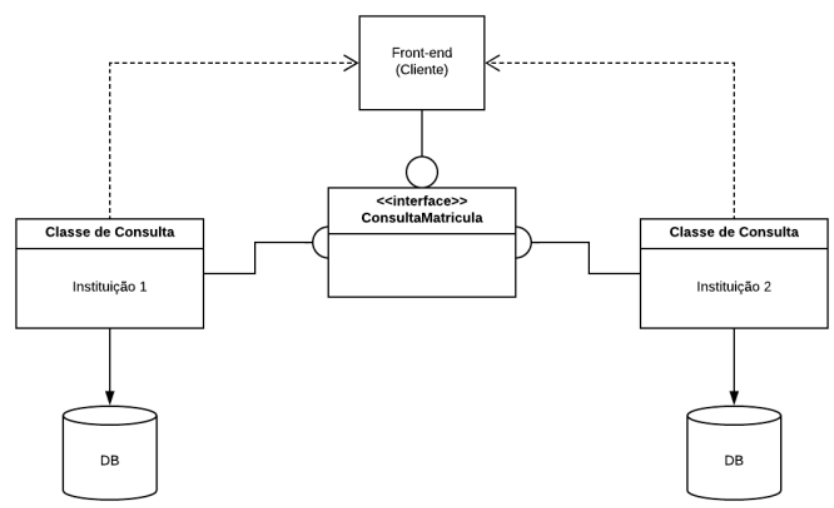

Figura 5.Arquitetura da aplicação EasyHalf. 
A ideia é que cada instituição de ensino que deseje utilizar a aplicação EasyHalf tenha uma classe específica que implemente a interface "ConsultaMatricula". Essa estratégia reduz o acoplamento entre os módulos da aplicação, de forma que essa classe possa ser implementada/modificada, sem que os demais componentes da aplicação sejam alterados.

\section{Avaliação da Aplicação EasyHalf}

Com as principais funcionalidades finalizadas, foi possível dar início à etapa de avaliação da aplicação EasyHalf. Tal avaliação foi realizada por meio de questionários, elaborados com base no modelo de aceitação de tecnologia (TAM - Technology Acceptance Model), que visaram a prover indícios sobre a utilidade e facilidade de uso percebida pelos usuários da aplicação (Davis, 1993).

O modelo TAM possui como objetivo explicar o comportamento das pessoas em relação à aceitação de uma tecnologia e tem sido utilizado em estudos recentes [Parreira Júnior e Penteado, 2018] para avaliação de produtos de software. Um dos constructos avaliados pelo modelo TAM é a utilidade percebida, que tenta medir o quanto uma pessoa acredita que usar determinada tecnologia aumenta sua produtividade. Para isso, sugere-se a criação de um questionário, ao qual são atribuídas afirmações relacionadas à utilidade percebida pelos usuários da tecnologia em análise. Para cada afirmação, o respondente deve escolher uma alternativa, conforme sua opinião sobre essa afirmação e de acordo com uma escala Likert.

O questionário aplicado aos estudantes foi divido em 5 seções, uma para levantar o perfil do estudante avaliador e quatro para avaliar diferentes aspectos da aplicação EasyHalf. O questionário foi aplicado a 7 (sete) estudantes de graduação da UFLA e os resultados das avaliações são apresentados nesta seção. Foi dada aos estudantes uma descrição do que eles poderiam fazer no momento na aplicação EasyHalf. Sabendo disso, os mesmos deveriam se cadastrar e, em seguida, responder às seguintes questões:

Q1: Em uma escala de 1 a 5, sendo um Muito Difícil e cinco Muito Fácil, descreva a facilidade de se descobrir quais são os estabelecimentos cadastrados no sistema. Em resposta a essa pergunta, 3 estudantes responderam que era muito fácil descobrir os estabelecimentos cadastrados; 3 responderam que era fácil; e 1 respondeu que era difícil.

Q2: Em uma escala de 1 a 5, sendo um Muito Difícil e cinco Muito Fácil, descreva a facilidade de se descobrir quais dos estabelecimentos cadastrados no sistema são autorizados por sua instituição de ensino. Em resposta a essa pergunta, 3 estudantes responderam que era muito fácil descobrir os estabelecimentos autorizados pela sua instituição; 3 responderam que era fácil; e 1 respondeu que era difícil.

Q3: Em uma escala de 1 a 5, sendo um Muito Difícil e cinco Muito Fácil, descreva a facilidade de se efetuar o cadastro no sistema. Em resposta a essa pergunta, 6 estudantes responderam que era muito fácil se cadastrar no sistema; e 1 respondeu que era fácil.

Q4: Em uma escala de 1 a 5, sendo um Muito Difícil e cinco Muito Fácil, descreva a facilidade em alterar suas informações de cadastro. Em resposta a essa pergunta, 6 
estudantes responderam que era muito fácil alterar suas informações; e 1 respondeu que era fácil.

Q5: Em uma escala de 1 a 5 sendo um Muito Difícil e cinco Muito Fácil, descreva a facilidade em ativar ou desativar sua conta. Em resposta a essa pergunta, todos os 7 estudantes responderam que era muito fácil ativar ou desativar sua conta.

Q6: Em uma escala de 1 a 5 sendo um Discordo totalmente e cinco Concordo totalmente, descreva se, na aplicação, é fácil encontrar as informações que deseja (por exemplo, onde me cadastrar, onde me autenticar e onde gerenciar minha conta). Em resposta a essa pergunta, todos os 7 estudantes responderam que concordavam totalmente.

Q7: Em uma escala de 1 a 5 sendo um Discordo totalmente e cinco Concordo totalmente, descreva se a aplicação EasyHalf possui visual (logotipo, layout dos menus, mensagens, entre outros) atraente. Em resposta a essa pergunta, 4 estudantes responderam que concordavam totalmente; 2 responderam que concordavam parcialmente; e 1 respondeu que não concordava nem discordava (neutro).

Q8: Em uma escala de 1 a 5 sendo um Discordo totalmente e cinco Concordo totalmente, após observar o que a aplicação EasyHalf oferece e qual o seu propósito, descreva se você usaria essa aplicação para acesso ao desconto de meia-entrada. Em resposta a essa pergunta, 6 estudantes responderam que concordavam totalmente e 1 respondeu que concordava parcialmente.

Q9: Em uma escala de 1 a 5 sendo um Discordo totalmente e cinco Concordo totalmente, descreva se você recomendaria o uso da aplicação EasyHalf. Em resposta a essa pergunta, 6 estudantes responderam que concordavam totalmente e 1 respondeu que concordava parcialmente.

Após finalizar as avaliações, foi possível observar que a ideia proposta pela aplicação EasyHalf foi bem aceita pelos estudantes avaliadores. Isso pode ser observado, principalmente, pelas respostas às perguntas Q4, Q5 e Q6. Também foi possível notar concordância dos avaliadores, observando as respostas dadas às perguntas que tratavam sobre possíveis melhorias trazidas pela aplicação EasyHalf ao processo atual de acesso ao desconto de meia-entrada.

Um dos aspectos negativos da aplicação, notado a partir da avaliação realizada, foi que a funcionalidade que permite aos usuários saber quais estabelecimentos estão cadastrados na aplicação e quais desses estabelecimentos estão autorizados pelas instituições precisa de aprimoramento. Essas foram as funções que tiveram pior avaliação pelos estudantes. Outro aspecto que não obteve uma avaliação convincente foi o layout da aplicação, pois alguns avaliadores consideraram que a aplicação precisa passar por ajustes em sua identidade visual. Por outro lado, as funções de cadastro e gerenciamento de contas obtiveram avaliações satisfatórias, nas quais a maioria dos estudantes considera fácil ou muito fácil realizar tais funções.

\section{Considerações Finais}

O presente trabalho propôs uma aplicação web, denominada EasyHalf, que permite, em tempo real, a consulta sobre a situação da matrícula de um estudante requerente do desconto de meia-entrada. Tal consulta é feita por meio do código de matrícula que o requerente do desconto possui. De acordo com o resultado, o estabelecimento pode 
tomar as decisões adequadas sobre o direito ou não de disponibilizar o desconto que foi requisitado.

A partir da avaliação realizada sobre a aplicação proposta, foi possível perceber que a ideia teve boa aceitação. Da mesma forma, foi possível perceber que existem melhorias a serem realizadas para que a aplicação atenda efetivamente as necessidades de seus usuários. Quanto às propostas de trabalhos futuros, uma das ideias é o desenvolvimento de um aplicativo móvel para simplificar ainda mais o processo de verificação da matrícula dos estudantes. A forma com que a situação da matrícula é consultada atualmente é por meio da digitação do código de matrícula fornecido pelo solicitante. Contudo, esse processo pode ser melhorado. A ideia é que os estudantes cadastrados na aplicação EasyHalf tenham um QR Code único, o qual poderia ser lido a partir de um dispositivo móvel, evitando assim a entrada manual de informações.

Outra proposta de trabalho futuro é estender a aplicação para que ela possa realizar a verificação dos casos de pessoas que não são estudantes, mas que também possuem direito ao desconto de meia-entrada, como por exemplo idosos e pessoas participantes de programas sociais do governo. Por fim, pretende-se realizar novos experimentos, com o intuito de aumentar a confiabilidade das afirmações realizadas neste texto, com relação à utilidade e facilidade de uso da aplicação EasyHalf.

\section{Referências}

Angular (2019). Disponível em: https://angular.io/. Acessado em: Mar/2020.

República Federativa do Brasil (2003) Lei no 12.993 de dezembro de 2013 - Seção 1. Disponível em: http://www.planalto.gov.br/ccivil_03/_ato20112014/2013/lei/112933.htm. Acessado em: Mar/2020.

Davis F.D. (1993) User acceptance of information technology: systemcharacteristics, user preceptions and behavioral impacts. Int J Man MachStud 38:475-48.

Gamma, E.; Helm R.; Johnson, R. e Vlissides, J (1995). Design Patterns: Elements of Reusable Object-Oriented Software. Addison-Wesley Longman Publishing Co., Inc., Boston, MA, USA.

Java (2019). Disponível em: https://www.java.com/pt_BR/download/. Acessado em: $\operatorname{Mar} / 2020$.

Parreira Júnior, Paulo Afonso e Penteado, Rosângela Aparecida Dellosso. ObasCId(Tool): an ontologically based approach for concern identification and classification and its computational support. Journal of the Brazilian Computer Society. 24. 10.1186/s13173-017-0067-6.

UNE. (2018). Carteira Nacional de Identificação Estudantil. https://www.documentodoestudante.com.br. 